Review Article

\title{
A Review on Approximation Results for Integral Operators in the Space of Functions of Bounded Variation
}

\author{
Laura Angeloni and Gianluca Vinti \\ Dipartimento di Matematica e Informatica, Università degli Studi di Perugia, Via Vanvitelli 1, 06123 Perugia, Italy \\ Correspondence should be addressed to Gianluca Vinti; gianluca.vinti@unipg.it
}

Received 16 May 2016; Accepted 20 July 2016

Academic Editor: Henryk Hudzik

Copyright (C) 2016 L. Angeloni and G. Vinti. This is an open access article distributed under the Creative Commons Attribution License, which permits unrestricted use, distribution, and reproduction in any medium, provided the original work is properly cited.

We present a review on recent approximation results in the space of functions of bounded variation for some classes of integral operators in the multidimensional setting. In particular, we present estimates and convergence in variation results for both convolution and Mellin integral operators with respect to the Tonelli variation. Results with respect to a multidimensional concept of $\varphi$-variation in the sense of Tonelli are also presented.

\section{Introduction}

The aim of the present paper is to give a review on recent results about convergence of integral operators of convolution type with respect to some concepts of multidimensional variation. We will consider the case of classical convolution integral operators of the form

$$
\left(T_{w} f\right)(\mathbf{s})=\int_{\mathbb{R}^{N}} K_{w}(\mathrm{t}) f(\mathbf{s}-\mathrm{t}) d \mathrm{t},
$$

$$
\mathbf{s} \in \mathbb{R}^{N}, w>0
$$

where $f \in L^{1}\left(\mathbb{R}^{N}\right)$ and $\left\{K_{w}\right\}_{w>0}$ is a family of approximate identities, as well as the case of Mellin integral operators of the form

$$
\left(M_{w} f\right)(\mathbf{s})=\int_{\mathbb{R}_{+}^{N}} K_{w}(\mathrm{t}) f(\mathrm{st})\langle\mathrm{t}\rangle^{-1} d \mathrm{t}
$$

where st $:=\left(s_{1} t_{1}, \ldots, s_{N} t_{N}\right)$, s, $\mathrm{t} \in \mathbb{R}_{+}^{N}$, and $\langle\mathrm{t}\rangle:=$ $\prod_{i=1}^{N} t_{i}$. The above operators (II) are of convolution type with respect to the homothetic operator and the measure $\mu(A)=\int_{A}(d \mathrm{x} /\langle\mathrm{x}\rangle)$, where $A$ is a Borel subset of $\mathbb{R}_{+}^{N}$ (which is an invariant measure with respect to the multiplicative operation).
An important tool in order to frame the results of the paper is the setting of the functional spaces we deal with. The BV-spaces, apart from the well-known importance from the mathematical point of view, also play an important role in problems of Image Reconstruction where some of the various approaches make use of integral operators of convolution type (see, e.g., sampling-type operators).

The working space will be the space of functions of bounded multidimensional variation in the sense of Tonelli (defined in Section 2) and, as further extension, in order to deal with a larger class of functions, we will consider the space $\mathrm{BV}^{\varphi}$, where $\varphi$ is a $\varphi$-function (see Section 2). We point out that, due to the necessary assumptions on the $\varphi$-function $\varphi$ (Assumption ii), the case of BV cannot be obtained as particular case of $\mathrm{BV}^{\varphi}$. This is the reason why the two settings have to be treated independently.

For the above classes of operators we will provide estimates, convergence results, and also a characterization of the absolutely ( $\varphi$-absolutely) continuous functions in terms of the respective convergence in variation. Moreover, the rate of approximation has been considered and examples of kernel functions to which the results can be applied are also furnished. Finally, also the nonlinear case for both the convolution and the Mellin-type operators has been considered.

Apart from the well-known importance of the classical convolution integral operators, the Mellin operators are very 
interesting and widely studied in approximation theory (for the basic theory see $[1,2]$ while, for results about similar homothetic-type operators, see, e.g., [3-16]), also because of their important applications in several fields. Among them, for example, we recall that Mellin analysis is deeply connected with some problems of Signal Processing, in particular with the so-called Exponential Sampling, which have applications in various problems of engineering and optical physics (see, e.g., $[17-20])$.

Concerning now the multidimensional concept of $\varphi$ variation introduced in [21], we point out that, due to the lack of an integral representation of the $\varphi$-variation for $\varphi$ absolutely continuous functions as happens for the classical variation, the major results about convergence require a different approach and suitable techniques. In particular, this holds for the convergence of the $\varphi$-modulus of continuity. Again, a similar problem occurs in case of Mellin integral operators on $\mathrm{BV}\left(\mathbb{R}_{+}^{N}\right)$, where the Tonelli integrals are defined via the log-measure. Moreover, in the latter case, in order to prove that the operators are absolutely continuous, in case of regular kernels (this is a crucial point to obtain the characterization of absolute continuity), one has to pass through an equivalent notion of absolute continuity (for the log-absolute continuity, see Section 4) compatible with the setting of $\mathbb{R}_{+}^{N}$ equipped with the log-measure.

We finally remark that, of course, all the results of the paper contain, in particular, the one-dimensional case (see [22-27]).

\section{Preliminaries and Some Concepts of Variation}

We will now recall the multidimensional concept of variation in the sense of Tonelli. Such definition was introduced by Tonelli [28] for functions of two variables and then extended to dimension $N>2$ by Radó [29] and Vinti [30].

Let us introduce some notations. If we are interested in the $j$ th coordinate of a vector $\mathrm{x}=\left(x_{1}, \ldots, x_{N}\right) \in \mathbb{R}^{N}$, we will write

$$
\begin{gathered}
\mathrm{x}_{j}^{\prime}=\left(x_{1}, \ldots, x_{j-1}, x_{j+1}, \ldots, x_{N}\right) \in \mathbb{R}^{N-1}, \\
\mathrm{x}=\left(\mathrm{x}_{j}^{\prime}, x_{j}\right),
\end{gathered}
$$

so that, for a function $f: \mathbb{R}^{N} \rightarrow \mathbb{R}$, there holds

$$
f(\mathrm{x})=f\left(\mathrm{x}_{j}^{\prime}, x_{j}\right) .
$$

Given an $N$-dimensional interval $I=\prod_{i=1}^{N}\left[a_{i}, b_{i}\right]$, by $I_{j}^{\prime}=$ $\left[\mathrm{a}_{j}^{\prime}, \mathrm{b}_{j}^{\prime}\right]$ we will denote the $(N-1)$-dimensional interval obtained by deleting the $j$ th coordinate from $I$; namely,

$$
I=\left[\mathrm{a}_{j}^{\prime}, \mathrm{b}_{j}^{\prime}\right] \times\left[a_{j}, b_{j}\right], \quad j=1, \ldots, N .
$$

Definition 1. A function $f: \mathbb{R}^{N} \rightarrow \mathbb{R}$ is said to be of bounded variation if the sections of $f$ are a.e. of bounded variation on $\mathbb{R}$ and their variation is summable; that is, $V_{\mathbb{R}}\left[f\left(\mathrm{x}_{j}^{\prime}, \cdot\right)\right]$ (the usual Jordan one-dimensional variation of the $j$ th section of $f)$ is finite a.e. $\mathrm{x}_{j}^{\prime} \in \mathbb{R}^{N-1}$ and $\int_{\mathbb{R}^{N-1}} V_{\mathbb{R}}\left[f\left(\mathrm{x}_{j}^{\prime}, \cdot\right)\right] d \mathrm{x}_{j}^{\prime}<+\infty$, for every $j=1, \ldots, N$.

In order to compute the variation of $f$ on an interval $I$, the first step is to define the $(N-1)$-dimensional integrals (the so-called Tonelli integrals)

$$
\Phi_{j}(f, I):=\int_{\mathrm{a}_{j}^{\prime}}^{\mathrm{b}_{j}^{\prime}} V_{\left[a_{j}, b_{j}\right]}\left[f\left(\mathrm{x}_{j}^{\prime}, \cdot\right)\right] d \mathrm{x}_{j}^{\prime}, \quad j=1, \ldots, N .
$$

Let now $\Phi(f, I)$ be the Euclidean norm of the vector $\left(\Phi_{1}(f, I), \ldots, \Phi_{N}(f, I)\right)$; that is,

$$
\Phi(f, I):=\left\{\sum_{j=1}^{N} \Phi_{j}^{2}(f, I)\right\}^{1 / 2}
$$

where we put $\Phi(f, I)=\infty$ if $\Phi_{j}(f, I)=\infty$ for some $j=$ $1, \ldots, N$

The variation of $f$ on an interval $I \subset \mathbb{R}^{N}$ is defined as

$$
V_{I}[f]:=\sup \sum_{k=1}^{m} \Phi\left(f, J_{k}\right)
$$

where the supremum is taken over all the families of $N$ dimensional intervals $\left\{J_{1}, \ldots, J_{m}\right\}$ which form partitions of $I$.

Finally, the variation of $f$ over the whole $\mathbb{R}^{N}$ is defined as

$$
V_{\mathbb{R}^{N}}[f]:=\sup _{I \subset \mathbb{R}^{N}} V_{I}[f]
$$

where the supremum is taken over all the intervals $I \subset \mathbb{R}^{N}$.

By

$$
\mathrm{BV}\left(\mathbb{R}^{N}\right):=\left\{f \in L^{1}\left(\mathbb{R}^{N}\right): V_{\mathbb{R}^{N}}[f]<+\infty\right\}
$$

we will denote the space of functions of bounded variation on $\mathbb{R}^{N}$.

We recall that it can be proved that if $f \in \mathrm{BV}\left(\mathbb{R}^{N}\right)$ then $\nabla f$ exists a.e. in $\mathbb{R}^{N}$ and $\nabla f \in L^{1}\left(\mathbb{R}^{N}\right)$ (see, e.g., $[29,30]$ ).

We point out that, in the multidimensional setting, it is natural to consider functions of bounded variation within the Lebesgue space $L^{1}\left(\mathbb{R}^{N}\right)$ : indeed this is analogous to the distributional concept of variation given by Cesari [31] and, in equivalent forms, by Krickeberg [32], De Giorgi [33], Giusti [34], and Serrin [35]. We notice that the definition of variation in the sense of Tonelli is equivalent to the distributional one in the class of functions which satisfy some approximate continuity properties (see, e.g., [30]).

We will now recall the concept of absolute continuity in sense of Tonelli.

Definition 2. A function $f: \mathbb{R}^{N} \rightarrow \mathbb{R}$ is locally absolutely continuous $\left(f \in \mathrm{AC}_{\text {loc }}\left(\mathbb{R}^{N}\right)\right)$ if, for every interval $I=$ $\prod_{i=1}^{N}\left[a_{i}, b_{i}\right]$ and for every $j=1,2, \ldots, N$, the $j$ th section $f\left(\mathrm{x}_{j}^{\prime}, \cdot\right):\left[a_{j}, b_{j}\right] \rightarrow \mathbb{R}$ is (uniformly) absolutely continuous for almost every $x_{j}^{\prime} \in\left[a_{j}^{\prime}, b_{j}^{\prime}\right]$. 
Similarly to the one-dimensional case, it is possible to prove that if $f \in \mathrm{BV}\left(\mathbb{R}^{N}\right) \cap \mathrm{AC}_{\text {loc }}\left(\mathbb{R}^{N}\right)$, then

$$
V_{\mathbb{R}^{N}}[f]=\int_{\mathbb{R}^{N}}|\nabla f(\mathrm{x})| d \mathrm{x}
$$

(see, e.g., $[29,30])$.

We will denote by $\mathrm{AC}\left(\mathbb{R}^{N}\right)$ the space of all the functions $f \in \mathrm{BV}\left(\mathbb{R}^{N}\right) \cap \mathrm{AC}_{\text {loc }}\left(\mathbb{R}^{N}\right)$.

In the following we will present also results about convergence for a family of Mellin integral operators. In order to study such kind of operators the most natural way is to consider $\left.\left.\mathbb{R}_{+}^{N}:=\right] 0,+\infty\right)^{N}$ (the domain of the functions where Mellin operators act), as a group with the multiplicative operation (instead of the additive operation on $\mathbb{R}^{N}$ ) and equipped with the logarithmic Haar-measure $\mu(A)=\int_{A}(d x /\langle x\rangle)(A$ is a Borel subset of $\mathbb{R}_{+}^{N}$ and $\langle\mathrm{x}\rangle:=\prod_{i=1}^{N} x_{i}, \mathrm{x}=\left(x_{1}, \ldots, x_{N}\right) \epsilon$ $\left.\mathbb{R}_{+}^{N}\right)$, instead of the usual Lebesgue measure. For this reason, in order to obtain results in BV-spaces for such kind of operators, it seems natural to adapt the definition of the Tonelli variation to this frame: we therefore introduced in [36] a new concept of multidimensional variation in which, in the Tonelli integrals, the Lebesgue measure is replaced by the logarithmic measure $\mu$.

Definition 3. One will say that $f \in \widetilde{L}^{1}\left(\mathbb{R}_{+}^{N}\right)$ is of bounded variation on $\mathbb{R}_{+}^{N}$ if the sections $f\left(\mathrm{x}_{j}^{\prime}, \cdot\right)$ are of bounded variation on $\mathbb{R}_{+}$a.e. $x_{j}^{\prime} \in \mathbb{R}_{+}^{N-1}$ and $V_{\mathbb{R}_{+}}\left[f\left(\mathrm{x}_{j}^{\prime}, \cdot\right)\right] \in \widetilde{L}^{1}\left(\mathbb{R}_{+}^{N-1}\right)$, where $\widetilde{L}^{1}\left(\mathbb{R}_{+}^{N}\right)$ denotes the space of the functions $f: \mathbb{R}_{+}^{N} \rightarrow \mathbb{R}$ such that $\int_{\mathbb{R}_{+}^{N}}^{+}|f(\mathrm{t})|\langle\mathrm{t}\rangle^{-1} d \mathrm{t}<+\infty$.

In order to define the multidimensional variation on $\mathbb{R}_{+}^{N}$, for a fixed interval $I:=\prod_{i=1}^{N}\left[a_{i}, b_{i}\right] \subset \mathbb{R}_{+}^{N}$ we consider the $(N-1)$-dimensional integrals

$$
\Phi_{j}(f, I):=\int_{\mathrm{a}_{j}^{\prime}}^{\mathrm{b}_{j}^{\prime}} V_{\left[a_{j}, b_{j}\right]}\left[f\left(\mathrm{x}_{j}^{\prime}, \cdot\right)\right] \frac{d \mathrm{x}_{j}^{\prime}}{\left\langle\mathrm{x}_{j}^{\prime}\right\rangle},
$$

where $\left\langle\mathrm{x}_{j}^{\prime}\right\rangle$ denotes the product $\prod_{i=1, i \neq j}^{N} x_{i}$. The remaining steps for the definition of the variation follow as before.

For the sake of simplicity, we will use the same notations for the variation in both the cases of $\mathbb{R}^{N}$ and $\mathbb{R}_{+}^{N}$ : as it is natural, when one works on $\mathbb{R}_{+}^{N}$, it is intended that the measure used is the logarithmic one.

The classical definition of Jordan variation [37] was extended in the literature in several directions: one of the first generalizations was the quadratic variation introduced by Wiener [38], extended to the $p$-variation, $p \geq 1[39,40]$, and later to the concept of $\varphi$-variation. The $\varphi$-variation was first introduced by Young [41] and then extensively studied by Musielak and Orlicz and their school (see, e.g., [22, 42-49]).

From now on we will assume that $\varphi: \mathbb{R}_{0}^{+} \rightarrow \mathbb{R}_{0}^{+}$is as follows.

Assumption i. $\varphi$ is a convex $\varphi$-function, where a $\varphi$-function is a continuous, nondecreasing function on $\mathbb{R}_{0}^{+}$, such that $\varphi(0)=0, \varphi(u)>0$ for $u>0$, and $\lim _{u \rightarrow+\infty} \varphi(u)=+\infty$.
Assumption ii. $u^{-1} \varphi(u) \rightarrow 0$ as $u \rightarrow 0^{+}$.

We recall that (see [22]) the $\varphi$-variation of $f: \mathbb{R} \rightarrow \mathbb{R}$ on $[a, b] \subset \mathbb{R}$ is defined as

$$
V_{[a, b]}^{\varphi}[f]:=\sup _{D} \sum_{i=1}^{n} \varphi\left(\left|f\left(s_{i}\right)-f\left(s_{i-1}\right)\right|\right),
$$

where the supremum is taken over all the partitions $D=\left\{s_{0}=\right.$ $\left.a, s_{1}, \ldots, s_{n}=b\right\}$ of the interval $[a, b]$, and

$$
V_{\mathbb{R}}^{\varphi}[f]:=\sup _{[a, b] \subset \mathbb{R}} V_{[a, b]}^{\varphi}[f] .
$$

Definition 4. A function $f: \mathbb{R} \rightarrow \mathbb{R}$ is said to be of bounded $\varphi$-variation $\left(f \in \mathrm{BV}^{\varphi}(\mathbb{R})\right)$ if $V_{\mathbb{R}}^{\varphi}[\lambda f]<+\infty$, for some $\lambda>0$.

The Musielak-Orlicz $\varphi$-variation was generalized to the multidimensional frame in [50] following the approach of Vitali. However, in order to study approximation problems, the approach of the Tonelli variation seems to be the most natural in this context. For such reason in [21] we introduced a concept of multidimensional $\varphi$-variation inspired by the Tonelli and C. Vinti approach.

Again, the crucial point is to define, for $j=1, \ldots, N$, the Tonelli integrals: in this case we put

$$
\Phi_{j}^{\varphi}(f, I):=\int_{\mathrm{a}_{j}^{\prime}}^{\mathrm{b}_{j}^{\prime}} V_{\left[a_{j}, b_{j}\right]}^{\varphi}\left[f\left(\mathrm{x}_{j}^{\prime}, \cdot\right)\right] d \mathrm{x}_{j}^{\prime},
$$

where $V_{\left[a_{j}, b_{j}\right]}^{\varphi}\left[f\left(\mathrm{x}_{j}^{\prime}, \cdot\right)\right]$ is the (one-dimensional) MusielakOrlicz $\varphi$-variation of the $j$ th section of $f$.

Putting now

$$
\Phi^{\varphi}(f, I):=\left\{\sum_{j=1}^{N}\left[\Phi_{j}^{\varphi}(f, I)\right]^{2}\right\}^{1 / 2},
$$

the multidimensional $\varphi$-variation of $f: \mathbb{R}^{N} \rightarrow \mathbb{R}$ on an interval $I \subset \mathbb{R}^{N}$ is defined as

$$
V_{I}^{\varphi}[f]:=\sup \sum_{k=1}^{m} \Phi^{\varphi}\left(f, J_{k}\right),
$$

(the supremum is taken over all the partitions $\left\{J_{1}, \ldots, J_{m}\right\}$ of I) and, finally,

$$
V_{\mathbb{R}^{N}}^{\varphi}[f]:=\sup _{I \subset \mathbb{R}^{N}} V_{I}^{\varphi}[f] .
$$

By

$$
\begin{aligned}
\operatorname{BV}^{\varphi} & \left(\mathbb{R}^{N}\right) \\
\quad & \left\{f \in L^{1}\left(\mathbb{R}^{N}\right): \exists \lambda>0 \text { s.t. } V^{\varphi}[\lambda f]<+\infty\right\}
\end{aligned}
$$

we will denote the space of functions of bounded $\varphi$-variation over $\mathbb{R}^{N}$.

Similarly to the classical variation, it is natural to introduce a concept of multidimensional $\varphi$-absolute continuity. 
Definition 5. One says that $f: \mathbb{R}^{N} \rightarrow \mathbb{R}$ is locally $\varphi$ absolutely continuous $\left(\mathrm{AC}_{\mathrm{loc}}^{\varphi}\left(\mathbb{R}^{N}\right)\right)$ if it is $\varphi$-absolutely continuous in the sense of Tonelli; that is, for every $I=$ $\prod_{i=1}^{N}\left[a_{i}, b_{i}\right] \subset \mathbb{R}^{N}$ and $j=1,2, \ldots, N$, the section $f\left(\mathrm{x}_{j}^{\prime}, \cdot\right)$ : $\left[a_{j}, b_{j}\right] \rightarrow \mathbb{R}$ is (uniformly) $\varphi$-absolutely continuous for almost every $\mathrm{x}_{j}^{\prime} \in\left[\mathrm{a}_{j}^{\prime}, \mathrm{b}_{j}^{\prime}\right]$.

Here (see [22]) a function $g:[a, b] \subset \mathbb{R} \rightarrow \mathbb{R}$ is $\varphi$ absolutely continuous if there exists $\lambda>0$ such that the following property holds:

for every $\varepsilon>0$, there exists $\delta>0$ for which

$$
\sum_{\nu=1}^{n} \varphi\left(\lambda\left|g\left(\beta^{\nu}\right)-g\left(\alpha^{\nu}\right)\right|\right)<\varepsilon
$$

for all the finite collections of nonoverlapping intervals $\left[\alpha^{\nu}, \beta^{\nu}\right] \subset[a, b], \nu=1, \ldots, n$, such that

$$
\sum_{\nu=1}^{n} \varphi\left(\beta^{\nu}-\alpha^{\nu}\right)<\delta
$$

By $\operatorname{AC}^{\varphi}\left(\mathbb{R}^{N}\right)$ we will denote the space of all the functions $f \in \mathrm{BV}^{\varphi}\left(\mathbb{R}^{N}\right) \cap \mathrm{AC}_{\text {loc }}^{\varphi}\left(\mathbb{R}^{N}\right)$.

As before, in order to obtain results for Mellin integral operators in $\mathrm{BV}^{\varphi}$-spaces in the multidimensional frame, we adapted the previous definition of $\varphi$-variation in the sense of Tonelli to the case of functions defined on $\mathbb{R}_{+}^{N}$ equipped with the logarithmic measure $\mu$. In such concept of multidimensional $\varphi$-variation, introduced in [51], the Tonelli integrals (13) are replaced by

$$
\Phi_{j}^{\varphi}(f, I):=\int_{\mathrm{a}_{j}^{\prime}}^{\mathrm{b}_{j}^{\prime}} V_{\left[a_{j}, b_{j}\right]}^{\varphi}\left[f\left(\mathrm{x}_{j}^{\prime}, \cdot\right)\right] \frac{d \mathrm{x}_{j}^{\prime}}{\left\langle\mathrm{x}_{j}^{\prime}\right\rangle} .
$$

Again, we will use the same notations, as in the case of the variation, for functions defined on both $\mathbb{R}^{N}$ and $\mathbb{R}_{+}^{N}$.

\section{Approximation Results for Convolution Integral Operators}

In this section we will present results about approximation in variation by means of the convolution integral operators, namely, (I), for $f \in L^{1}\left(\mathbb{R}^{N}\right)$. Here $\left\{K_{w}\right\}_{w>0}$ is a family of approximate identities (see, e.g., [52]); that is,

$\left(K_{w} .1\right) K_{w}: \mathbb{R}^{N} \rightarrow \mathbb{R}$ is a measurable essentially bounded function such that $K_{w} \in L^{1}\left(\mathbb{R}^{N}\right),\left\|K_{w}\right\|_{1} \leq$ $A$ for an absolute constant $A>0$ and $\int_{\mathbb{R}^{N}} K_{w}(\mathrm{t}) d \mathrm{t}=$ 1 , for every $w>0$;

$\left(K_{w} .2\right)$ for any fixed $\delta>0, \int_{|\mathrm{t}|>\delta}\left|K_{w}(\mathrm{t})\right| d \mathrm{t} \rightarrow 0$, as $w \rightarrow+\infty$.

In the following we will say that $\left\{K_{w}\right\}_{w>0} \subset \mathscr{K}_{w}$ if $\left(K_{w} .1\right)$ and $\left(K_{w} .2\right)$ are satisfied.
Of course the operators (I) are well-defined for every $f \in$ $L^{1}\left(\mathbb{R}^{N}\right)$ and therefore in particular for every function $f \in$ $\mathrm{BV}\left(\mathbb{R}^{N}\right)$, since

$$
\left|\left(T_{w} f\right)(\mathrm{x})\right| \leq\|f\|_{1}\left\|K_{w}\right\|_{\infty}, \quad \forall \mathrm{x} \in \mathbb{R}^{N} .
$$

We first recall that the family of operators (I) map $\mathrm{BV}\left(\mathbb{R}^{N}\right)$ into itself. Indeed, the following estimate holds.

Proposition 6 (see [25]). Let $f \in B V\left(\mathbb{R}^{N}\right)$. If $\left\{K_{w}\right\}_{w>0}$ satisfies $\left(K_{w} .1\right)$, then

$$
V_{\mathbb{R}^{N}}\left[T_{w} f\right] \leq A V_{\mathbb{R}^{N}}[f],
$$

$w>0$, where $A$ is the constant of Assumption $\left(K_{w} .1\right)$.

Remark 7. In the case of nonnegative kernels $\left\{K_{w}\right\}_{w>0}$, Proposition 6 gives the "variation diminishing property" for the operators $T_{w} f$ : indeed in this case $A=\left\|K_{w}\right\|_{1}=1, w>0$.

In order to obtain the main result about convergence in variation, the following estimate of the error of approximation is essential.

Proposition 8 (see [25]). If $f \in B V\left(\mathbb{R}^{N}\right)$ then, for every $w>$ 0 ,

$$
\begin{aligned}
& V_{\mathbb{R}^{N}} {\left[T_{w} f-f\right] } \\
& \quad \leq \int_{\mathbb{R}^{N}} V_{\mathbb{R}^{N}}[f(\cdot-\mathrm{t})-f(\cdot)]\left|K_{w}(\mathrm{t})\right| d \mathrm{t} .
\end{aligned}
$$

Another important tool is a characterization of the convergence for the modulus of smoothness of $f$, defined as

$$
\omega(f, \delta):=\sup _{|\mathrm{t}| \leq \delta} V_{\mathbb{R}^{N}}\left[\tau_{\mathrm{t}} f-f\right],
$$

where $\left(\tau_{\mathrm{t}} f\right)(\mathrm{s}):=f(\mathrm{~s}-\mathrm{t})$, for every $\mathrm{s}, \mathrm{t} \in \mathbb{R}^{N}$, is the translation operator (see, e.g., $[6,53])$.

Theorem 9 (see [25]). Let $f \in B V\left(\mathbb{R}^{N}\right)$. Then $f \in A C\left(\mathbb{R}^{N}\right)$ if and only if

$$
\lim _{\delta \rightarrow 0} \omega(f, \delta)=0
$$

The proof of the sufficient part of this result is a consequence of integral representation (9) of the variation for absolutely continuous functions and of the continuity in $L^{1}$ of the translation operator. For the necessary part, in [25] it is proved that if the kernel functions $K_{w}$ are absolutely continuous (as it happens in the most common cases), then also the integral operators $T_{w} f$ belong to $\operatorname{AC}\left(\mathbb{R}^{N}\right)$. Then, since $\mathrm{AC}\left(\mathbb{R}^{N}\right)$ is a closed subspace of $\mathrm{BV}\left(\mathbb{R}^{N}\right)$ with respect to the convergence in variation [25] and by estimate (23), in case of regular kernels, the convergence of the modulus of smoothness implies that $f \in \operatorname{AC}\left(\mathbb{R}^{N}\right)$.

By means of Proposition 8 and Theorem 9 it is possible to obtain the main result about convergence for absolutely continuous functions. 
Theorem 10 (see [25]). If $f \in A C\left(\mathbb{R}^{N}\right)$ and $\left\{K_{w}\right\}_{w>0} \subset \mathscr{K}_{w}$, then

$$
\lim _{w \rightarrow+\infty} V_{\mathbb{R}^{N}}\left[T_{w} f-f\right]=0 .
$$

Remark 11. We point out that the assumption of absolute continuity of the function is crucial to obtain the main convergence theorem and such result does not hold, in general, if, for example, $f \in \mathrm{BV}\left(\mathbb{R}^{N}\right) \backslash \mathrm{AC}\left(\mathbb{R}^{N}\right)$. For example, in the case $N=1$, let us consider $f: \mathbb{R} \rightarrow \mathbb{R}$ defined by

$$
f(x)= \begin{cases}1, & |x| \leq 1, \\ 0, & |x|>1 .\end{cases}
$$

First of all we point out that $V\left[\tau_{t} f-f\right] \nrightarrow 0$ as $t \rightarrow 0$. Let us now consider the Poisson-Cauchy kernel defined as

$$
K_{w}(t)=\sqrt{\frac{2}{\pi}} \frac{w}{1+w^{2} t^{2}}, \quad w>0, t \in \mathbb{R} .
$$

Then

$$
\begin{aligned}
& \left(T_{w} f\right)(s) \\
& \quad=\sqrt{\frac{2}{\pi}}[\arctan (w(s+1))-\arctan (w(s-1))],
\end{aligned}
$$

$s \in \mathbb{R}$,

and therefore

$$
\begin{aligned}
V_{\mathbb{R}}\left[T_{w} f-f\right] & \geq V_{(-\infty,-1]}\left[T_{w} f-f\right] \\
& =\left|\left(T_{w} f\right)(-1)-\lim _{s \rightarrow-\infty}\left(T_{w} f\right)(s)\right| \\
& =-\sqrt{\frac{2}{\pi}} \arctan (-2 w) .
\end{aligned}
$$

This implies that

$$
\liminf _{w \rightarrow+\infty} V_{\mathbb{R}}\left[T_{w} f-f\right] \geq \sqrt{\frac{\pi}{2}},
$$

and hence $V_{\mathbb{R}}\left[T_{w} f-f\right] \nrightarrow 0$ as $w \rightarrow+\infty$.

In case of regular kernels, by the closure of $\mathrm{AC}\left(\mathbb{R}^{N}\right)$ in $\mathrm{BV}\left(\mathbb{R}^{N}\right)$, the converse of Theorem 10 is also true. Hence we obtain the following characterization of the space of the absolutely continuous functions, similarly to what happens in the one-dimensional case for the Jordan variation.

Theorem 12 (see [25]). Assume that $f \in B V\left(\mathbb{R}^{N}\right)$ and $\left\{K_{w}\right\}_{w>0} \subset \mathscr{K}_{w} \cap A C\left(\mathbb{R}^{N}\right)$. Then $f \in A C\left(\mathbb{R}^{N}\right)$ if and only if

$$
\lim _{w \rightarrow+\infty} V_{\mathbb{R}^{N}}\left[T_{w} f-f\right]=0 .
$$

The previous results were generalized to the case of the multidimensional $\varphi$-variation in [21]. In particular, besides a kind of variation diminishing property, in [21] the following estimate for the error of approximation is obtained.
Proposition 13 (see [21]). Let $f \in B V^{\varphi}\left(\mathbb{R}^{N}\right)$ and let $\left\{K_{w}\right\}_{w>0}$ be such that $\left(K_{w} .1\right)$ is satisfied. Then, for every $\lambda, \delta>0$ and for every $w>0$,

$$
\begin{aligned}
V_{\mathbb{R}^{N}}^{\varphi} & {\left[\lambda\left(T_{w} f-f\right)\right] } \\
\leq & A^{-1}\left\{\omega^{\varphi}(\lambda A f, \delta) \int_{|\mathrm{t}| \leq \delta}\left|K_{w}(\mathrm{t})\right| d \mathrm{t}\right. \\
+ & \left.V_{\mathbb{R}^{N}}^{\varphi}[2 \lambda A f] \int_{|\mathrm{t}|>\delta}\left|K_{w}(\mathrm{t})\right| d \mathrm{t}\right\},
\end{aligned}
$$

where $\omega^{\varphi}(f, \delta):=\sup _{|\mathrm{t}| \leq \delta} V_{\mathbb{R}^{N}}^{\varphi}\left[\tau_{\mathrm{t}} f-f\right]$ is the $\varphi$-modulus of smoothness of $f$.

Using the previous estimate, the main convergence result follows by the singularity assumption on the kernel functions $\left(K_{w}, 2\right)$ and by the convergence for the $\varphi$-modulus of smoothness.

Theorem 14 (see [54]). Let $f \in B V^{\varphi}\left(\mathbb{R}^{N}\right)$. Then there exists $\lambda>0$ such that

$$
\lim _{\delta \rightarrow 0^{+}} \omega^{\varphi}(\lambda f, \delta)=0,
$$

if and only if $f \in A C_{\mathrm{loc}}^{\varphi}\left(\mathbb{R}^{N}\right)$.

The convergence for the modulus of smoothness, in case of the Tonelli variation, is a direct consequence of the integral representation of the variation for absolutely continuous functions (9). On the contrary, for the $\varphi$-variation there are no results of this kind and, in order to get the convergence in $\varphi$-variation of the $\varphi$-modulus of smoothness, it is necessary to use a different technique. In particular (see [54]), the crucial point is to construct a kind of "step" functions that approximate the function $f$ and for which a convergence result can be proved.

Using Theorem 14, it is possible to obtain the main result of convergence in $\varphi$-variation for the convolution integral operators (I).

Theorem 15 (see [21]). If $f \in A C^{\varphi}\left(\mathbb{R}^{N}\right)$ and $\left\{K_{w}\right\}_{w>0} \subset \mathscr{K}_{w}$, then there exists $\lambda>0$ such that

$$
\lim _{w \rightarrow+\infty} V_{\mathbb{R}^{N}}^{\varphi}\left[\lambda\left(T_{w} f-f\right)\right]=0 .
$$

To give a sketch of the proof, the starting point is the estimate of Proposition 13 for the error of approximation $V_{\mathbb{R}^{N}}^{\varphi}\left[\lambda\left(T_{w} f-f\right)\right]$. By Theorem 14, we have that $\omega^{\varphi}(\lambda f, \delta)$ tends to 0 , for sufficiently small $\delta>0$, while, by Assumption $\left(K_{w} .2\right)$ on the kernel functions, in correspondence with such small $\delta, \int_{|t|>\delta}\left|K_{w}(\mathrm{t})\right| d \mathrm{t}$ converges to 0 for $w$ large enough; hence the result follows, taking into account $\left(K_{w} \cdot 1\right)$ and the fact that $f \in \mathrm{BV}^{\varphi}\left(\mathbb{R}^{N}\right)$.

As before, in case of regular kernels the converse of Theorem 15 is also true.

Theorem 16 (see [21]). Let $f \in B V^{\varphi}\left(\mathbb{R}^{N}\right)$ and let $\left\{K_{w}\right\}_{w>0} \subset$ $\mathscr{K}_{w} \cap A C^{\varphi}\left(\mathbb{R}^{N}\right)$. Then $f \in A C^{\varphi}\left(\mathbb{R}^{N}\right)$ if and only if there exists $\lambda>0$ such that

$$
\lim _{w \rightarrow+\infty} V_{\mathbb{R}^{N}}^{\varphi}\left[\lambda\left(T_{w} f-f\right)\right]=0 .
$$


Remark 17. We point out that it is easy to find examples of kernel functions to which the previous results can be applied. Among them, for example, the Gauss-Weierstrass kernel is defined as

$$
G_{w}(t)=\frac{w^{N}}{\pi^{N / 2}} e^{-w^{2}|t|^{2}}, \quad t \in \mathbb{R}^{N}, w>0,
$$

or the Picard kernel

$$
P_{w}(\mathrm{t})=\frac{w^{N} \Gamma(N / 2)}{2 \pi^{N / 2} \Gamma(N)} e^{-w|\mathrm{t}|}, \quad \mathrm{t} \in \mathbb{R}^{N}, w>0,
$$

where $\Gamma$ is the Gamma Euler function (see Figure 1).

\section{Approximation Results for Mellin Integral Operators}

We now turn our attention to Mellin integral operators, defined as (II), where st $:=\left(s_{1} t_{1}, \ldots, s_{N} t_{N}\right)$, s, $t \in \mathbb{R}_{+}^{N}$. On the kernel functions $\left\{K_{w}\right\}_{w>0}$ we assume that

$\left(\widetilde{K}_{w} .1\right) K_{w}: \mathbb{R}_{+}^{N} \rightarrow \mathbb{R}$ is a measurable essentially bounded function such that $K_{w} \in \widetilde{L}^{1}\left(\mathbb{R}_{+}^{N}\right)$, $\left\|K_{w}\right\|_{\widetilde{L}^{1}} \leq A$ for an absolute constant $A>0$ and $\int_{\mathbb{R}_{+}^{N}} K_{w}(\mathrm{t})\langle\mathrm{t}\rangle^{-1} d \mathrm{t}=1$, for every $w>0$;

$\left(\widetilde{K}_{w} .2\right)$ for every fixed $0<\delta<1$, $\int_{|1-\mathrm{t}|>\delta}\left|K_{w}(\mathrm{t})\right|\langle\mathrm{t}\rangle^{-1} d \mathrm{t} \rightarrow 0$, as $w \rightarrow+\infty$,

that is, the assumptions of approximate identities, adapted to the present setting of $\mathbb{R}_{+}^{N}$. If $\left\{K_{w}\right\}_{w>0}$ satisfy $\left(\widetilde{K}_{w} \cdot 1\right)$ and $\left(\widetilde{K}_{w} .2\right)$, we will write $\left\{K_{w}\right\}_{w>0} \subset \widetilde{\mathscr{K}}_{w}$.

For Mellin integral operators it is possible to develop an "approximation theory" similar to the case of the convolution integral operators; however, one of the main differences is the homothetic structure of $\mathbb{R}_{+}^{N}$ which leads to the choice of the logarithmic measure $\mu$ and also to the necessity to adapt some definitions. For example, the modulus of smoothness of $f \in$ $\mathrm{BV}\left(\mathbb{R}_{+}^{N}\right)$ has to be now defined as

$$
\omega(f, \delta):=\sup _{|\mathbf{1}-\mathrm{t}| \leq \delta} V_{\mathbb{R}_{+}^{N}}\left[\sigma_{\mathrm{t}} f-f\right],
$$

$0<\delta<1$, where $\left(\sigma_{\mathrm{t}} f\right)(\mathrm{s}):=f(\mathrm{st})$, for every $\mathrm{s}, \mathrm{t} \in \mathbb{R}_{+}^{N}$, is the homothetic operator and $\mathbf{1}=(1, \ldots, 1)$ is the unit vector of $\mathbb{R}_{+}^{N}$. Such notion of modulus of smoothness is the natural generalization, in the present frame of $\mathrm{BV}\left(\mathbb{R}_{+}^{N}\right)$, of the classical modulus of continuity (see, e.g., $[6,21,25])$.

Of course, due to the presence of the logarithmic measure, we cannot use the integral representation of the Tonelli variation; nevertheless, it is possible to directly prove a result of convergence in variation for the modulus of smoothness in case of AC-functions, using a kind of "separated" variations $\left(V^{j}[f], j=1, \ldots, N\right)$ which take into account just a single direction instead of all the $N$ directions.

Theorem 18 (see [36]). If $f \in A C\left(\mathbb{R}_{+}^{N}\right)$, then

$$
\lim _{\delta \rightarrow 0^{+}} \omega(f, \delta)=0 \text {. }
$$

By means of the previous result and an estimate for the error of approximation $\left(M_{w} f-f\right)$ analogous to Proposition 13 , it is possible to prove the following convergence result.

Theorem 19. Let $f \in A C\left(\mathbb{R}_{+}^{N}\right)$ and $\left\{K_{w}\right\}_{w>0} \subset \widetilde{\mathscr{K}}_{w}$. Then

$$
\lim _{w \rightarrow+\infty} V_{\mathbb{R}_{+}^{N}}\left[M_{w} f-f\right]=0 .
$$

A natural question now is whether, at least in case of ACkernels, the converse of the previous result holds, as in the case of convolution operators. Actually, due to the form of the operators $M_{w} f$, such question is now much more delicate and direct approach cannot be used. In order to solve the problem, it is necessary to use another concept of absolute continuity (the log-absolute continuity), equivalent to the classical one, which takes into account the logarithmic measure $\mu$. We first present the definition in the one-dimensional case.

Definition 20 (see [55]). One says that $f: \mathbb{R}_{+} \rightarrow \mathbb{R}$ is logabsolutely continuous on $[a, b] \subset \mathbb{R}_{+}\left(f \in \mathrm{AC}_{\log }([a, b])\right)$ if for every $\varepsilon>0$ there exists $\delta>0$ such that, for every collection of nonoverlapping intervals $\left[\alpha^{\nu}, \beta^{\nu}\right]_{\nu=1}^{n}$ in $[a, b]$ such that

$$
\sum_{\nu=1}^{n}\left|\log \left(\beta^{\nu}\right)-\log \left(\alpha^{\nu}\right)\right|<\delta
$$

then

$$
\sum_{\nu=1}^{n}\left|f\left(\beta^{\nu}\right)-f\left(\alpha^{\nu}\right)\right|<\varepsilon
$$

By $\mathrm{AC}_{\log }\left(\mathbb{R}_{+}\right)$we will denote the space of functions which are of bounded variation on $\mathbb{R}_{+}$and log-absolutely continuous on $[a, b]$, for every $[a, b] \subset \mathbb{R}_{+}$.

Now, in the general multidimensional frame, $f: \mathbb{R}_{+}^{N} \rightarrow$ $\mathbb{R}$ is log-absolutely continuous on $I=\prod_{i=1}^{N}\left[a_{i}, b_{i}\right] \subset \mathbb{R}_{+}^{N}$ if, for every $j=1,2, \ldots, N$, the $j$ th sections of $f, f\left(\mathrm{x}_{j}^{\prime}, \cdot\right):\left[a_{j}, b_{j}\right] \rightarrow$ $\mathbb{R}$, are (uniformly) log-absolutely continuous for almost every $\mathrm{x}_{j}^{\prime} \in \mathbb{R}_{+}^{N-1}$.

By means of the definition of the log-absolute continuity, in [55] it is proved that Mellin integral operators, as the classical convolution operators, preserve absolute continuity: this, together with the fact that the set of the absolutely continuous functions is a closed subspace of the set of the BVfunctions, allows us to obtain the following characterization.

Theorem 21 (see [55]). Let $f \in B V\left(\mathbb{R}_{+}^{N}\right)$ and $\left\{K_{w}\right\}_{w>0} \subset \widetilde{\mathscr{K}}_{w} \cap$ $A C\left(\mathbb{R}_{+}^{N}\right)$. Then $f \in A C\left(\mathbb{R}_{+}^{N}\right)$ if and only if $\lim _{w \rightarrow+\infty} V_{\mathbb{R}_{+}^{N}}\left[M_{w} f\right.$ $-f]=0$.

In [56] approximation properties for Mellin integral operators were studied in the frame of $\mathrm{BV}^{\varphi}$-spaces, using the multidimensional version of the $\varphi$-variation on $\mathbb{R}_{+}^{N}$ introduced in [51]. In particular the following theorem is obtained.

Theorem 22 (see [56]). Let $f \in A C^{\varphi}\left(\mathbb{R}_{+}^{N}\right)$ and $\left\{K_{w}\right\}_{w>0} \subset$ $\widetilde{\mathscr{K}}_{w}$. Then there exists a constant $\mu>0$ such that

$$
\lim _{w \rightarrow+\infty} V_{\mathbb{R}_{+}^{N}}^{\varphi}\left[\mu\left(M_{w} f-f\right)\right]=0 .
$$




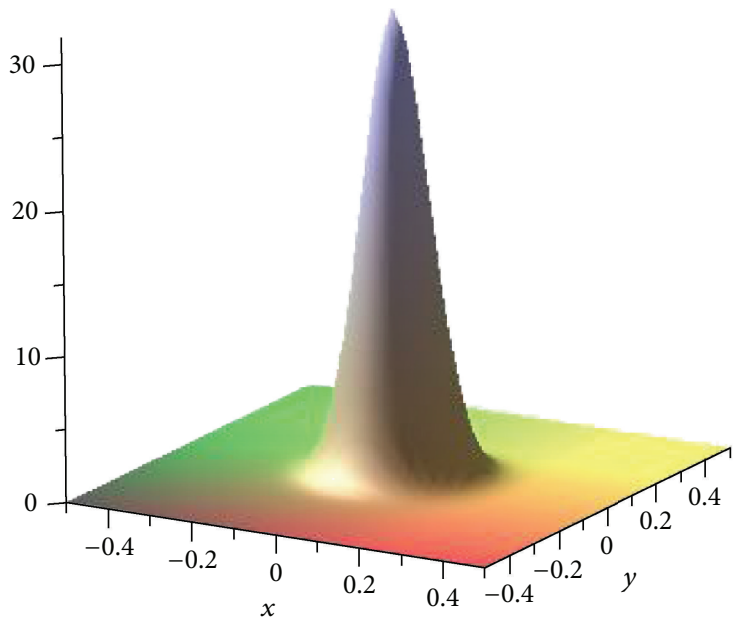

(a)

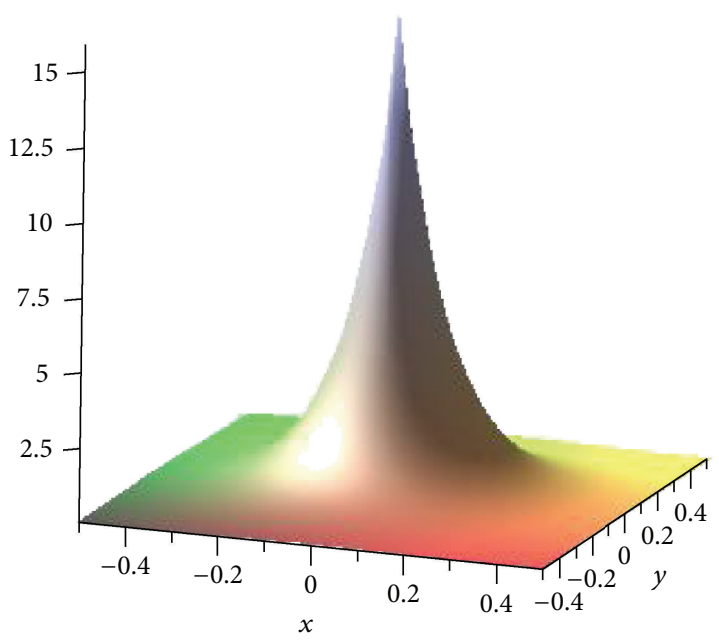

(b)

Figure 1: Examples of Gauss-Weierstrass kernel $G_{w}(x, y)$ and Picard kernel $P_{w}(x, y)$ with $w=10$.

The proof of Theorem 22 is based on the estimate of the error of approximation [56],

$$
\begin{aligned}
V^{\varphi}[ & \left.\lambda\left(M_{w} f-f\right)\right] \\
\leq & \omega^{\varphi}(\lambda A f, \delta) \\
& +A^{-1} V^{\varphi}[2 \lambda A f] \int_{|1-\mathrm{t}|\rangle \delta}\left|K_{w}(\mathrm{t})\right|\langle\mathrm{t}\rangle^{-1} d \mathrm{t},
\end{aligned}
$$

and on a convergence result for the $\varphi$-modulus of smoothness

$$
\omega^{\varphi}(f, \delta):=\sup _{|1-t| \leq \delta} V_{\mathbb{R}_{+}^{N}}^{\varphi}\left[\sigma_{\mathrm{t}} f-f\right],
$$

$\delta>0$. This last result was obtained in [51] by means of a direct approach: the function $f$ is approximated in $\varphi$-variation by two auxiliary functions, constructed on a grid on which their sections are piecewise constant.

In order to prove the converse of Theorem 22, it is again necessary to use a concept of logarithmic $\varphi$-absolute continuity, which takes into account the homothetic structure of $\mathbb{R}_{+}^{N}$. We report below the definition in the one-dimensional case, while for the multidimensional case it is sufficient to proceed as for the log-absolute continuity.

Definition 23 (see [57]). One says that $f:[a, b] \rightarrow \mathbb{R}$ is $\log$ $\varphi$-absolutely continuous on $[a, b] \subset \mathbb{R}_{+}$if there exists $\lambda>0$ such that, for every $\varepsilon>0$, there exists $\delta>0$ for which

$$
\sum_{\nu=1}^{n} \varphi\left(\lambda\left|f\left(\beta^{\nu}\right)-f\left(\alpha^{\nu}\right)\right|\right)<\varepsilon
$$

for all finite collections of nonoverlapping intervals $\left[\alpha^{\nu}, \beta^{\nu}\right] \mathrm{C}$ $[a, b], v=1, \ldots, n$, such that

$$
\sum_{\nu=1}^{n} \varphi\left(\log \left(\beta^{\nu}\right)-\log \left(\alpha^{\nu}\right)\right)<\delta .
$$

The $\log -\varphi$-absolute continuity is equivalent to the $\varphi$ absolute continuity and allows obtaining the characterization of $\mathrm{AC}^{\varphi}\left(\mathbb{R}_{+}^{N}\right)$ in terms of convergence in $\varphi$-variation of Mellin integral operators.

Theorem 24 (see [57]). Let $f \in B V^{\varphi}\left(\mathbb{R}_{+}^{N}\right)$ and $\left\{K_{w}\right\}_{w>0} \subset$ $\widetilde{\mathscr{K}}_{w} \cap A C^{\varphi}\left(\mathbb{R}_{+}^{N}\right)$. Then $f \in A C^{\varphi}\left(\mathbb{R}_{+}^{N}\right)$ if and only if there exists $\lambda>0$ such that $\lim _{w \rightarrow+\infty} V_{\mathbb{R}^{N}}^{\varphi}\left[\lambda\left(M_{w} f-f\right)\right]=0$.

Remark 25. We point out that taking $N=1$ as particular case of Theorem 24 we obtain the characterization of $\varphi$ absolute continuity in the one-dimensional case, namely, for the classical Musielak-Orlicz $\varphi$-variation.

Remark 26. It is not difficult to find examples of kernel functions which fulfill Assumptions $\left(\widetilde{K}_{w} \cdot 1\right)$ and $\left(\widetilde{K}_{w} \cdot 2\right)$. Among them, for example, the moment-type kernels (or average kernels) are defined as

$$
A_{w}(\mathrm{t}):=w^{N}\langle\mathrm{t}\rangle^{w} \chi_{] 0,1\left[^{N}\right.}(\mathrm{t}), \quad \mathrm{t} \in \mathbb{R}_{+}^{N}, w>0 .
$$

It is easy to see that they fulfill Assumption $\left(\widetilde{K}_{w} \cdot 1\right)$. Moreover, for every $\delta \in] 0,1[,|\mathbf{1}-\mathrm{t}|>\delta$ implies that there exists an index $j=1, \ldots, N$ such that $\left|1-t_{j}\right|>\delta / \sqrt{N}$; hence $\{\mathrm{t} \epsilon$ ] $0,1\left[{ }^{N}:|1-t|>\delta\right\} \subset \bigcup_{j=1}^{N}\left\{\mathrm{t} \in \mathbb{R}_{+}^{N}: 0<t_{j}<1-\delta / \sqrt{N}, 0<\right.$ $\left.t_{i}<1, \forall i \neq j\right\}$. Therefore

$$
\begin{aligned}
& \int_{|1-\mathrm{t}|>\delta}\left|A_{w}(\mathrm{t})\right|\langle\mathrm{t}\rangle^{-1} d \mathrm{t} \\
& \leq \sum_{j=1}^{N}\left\{\left(\prod_{i \neq j} \int_{0}^{1} w t_{i}^{w-1} d t_{i}\right) \int_{0}^{1-\delta / \sqrt{N}} w t_{j}^{w-1} d t_{j}\right\} \\
& =N\left(1-\frac{\delta}{\sqrt{N}}\right)^{w} \longrightarrow 0,
\end{aligned}
$$

as $w \rightarrow+\infty$; that is, also $\left(\widetilde{K}_{w} .2\right)$ is satisfied. 
Other families of kernel functions to which the previous results can be applied are the Mellin-Gauss-Weierstrass kernels, defined as

$$
\widetilde{G}_{w}(\mathrm{t}):=\frac{w^{N}}{\pi^{N / 2}} e^{-w^{2}|\log t|^{2}}, \quad \mathrm{t} \in \mathbb{R}_{+}^{N}, w>0,
$$

or the Mellin-Picard kernels, which are defined as

$$
\widetilde{P}_{w}(\mathrm{t}):=\frac{w^{N}}{2 \pi^{N / 2}} \frac{\Gamma(N / 2)}{\Gamma(N)} e^{-w|\log \mathrm{t}|}, \quad \mathrm{t} \in \mathbb{R}_{+}^{N}, w>0 .
$$

We point out that these definitions are the natural reformulations, in the present multiplicative setting of $\mathbb{R}_{+}^{N}$, of the classical Gauss-Weierstrass kernels and Picard kernels, respectively (see Remark 17).

\section{Further Results}

We will now give some hints about further approximation results that were obtained in $\mathrm{BV}$-spaces.

First of all, an interesting problem is to study the nonlinear versions of operators (I) and (II). We point out that the nonlinear case is much more delicate than the linear one and requires some ad hoc assumptions; on the other side, it not only is interesting from a mathematical point of view, being of course more general than the linear one, but also is important from the point of view of the applications. Indeed, there are several applicative problems that cannot be faced by means of linear processes; an example is furnished by some problems of Signal Processing.

The nonlinear version of the convolution integral operators (II) is

$$
\begin{aligned}
\left(\bar{T}_{w} f\right)(\mathrm{s})=\int_{\mathbb{R}^{N}} \bar{K}_{w}(\mathrm{t}, f(\mathrm{~s}-\mathrm{t})) d \mathrm{t}, & \\
& w>0, \mathbf{s} \in \mathbb{R}^{N},
\end{aligned}
$$

where $\left\{\bar{K}_{w}\right\}_{w>0}$ is a family of measurable functions $\bar{K}_{w}: \mathbb{R}^{N} \times$ $\mathbb{R} \rightarrow \mathbb{R}$ of the form

$$
\bar{K}_{w}(\mathrm{t}, u)=L_{w}(\mathrm{t}) H_{w}(u)
$$

for every $t \in \mathbb{R}^{N}, u \in \mathbb{R}$. Here $L_{w}: \mathbb{R}^{N} \rightarrow \mathbb{R}$ and $H_{w}: \mathbb{R} \rightarrow$ $\mathbb{R}$ with $H_{w}(0)=0$ is a Lipschitz kernel for every $w>0$; that is, there exists $K>0$ such that

$$
\left|H_{w}(u)-H_{w}(v)\right| \leq K|u-v|, \quad \forall u, v \in \mathbb{R} .
$$

Moreover we assume that

$\left(\bar{K}_{w} .1\right) L_{w}: \mathbb{R}^{N} \rightarrow \mathbb{R}$ is a measurable function such that $L_{w} \in L^{1}\left(\mathbb{R}^{N}\right),\left\|L_{w}\right\|_{1} \leq A$, for some $A>0$ and for every $w>0$, and $\int_{\mathbb{R}^{N}} L_{w}(\mathrm{t}) d \mathrm{t}=1$, for every $w>0$;

$\left(\bar{K}_{w} .2\right)$ for any fixed $\delta>0, \int_{|t|>\delta}\left|L_{w}(\mathrm{t})\right| d \mathrm{t} \rightarrow 0$, as $w \rightarrow+\infty$;

$\left(\bar{K}_{w} .3\right)$ denoted by $G_{w}(u):=H_{w}(u)-u, u \in \mathbb{R}, w>0$,

$$
\frac{V_{J}\left[G_{w}\right]}{m(J)} \longrightarrow 0, \quad \text { as } w \longrightarrow+\infty
$$

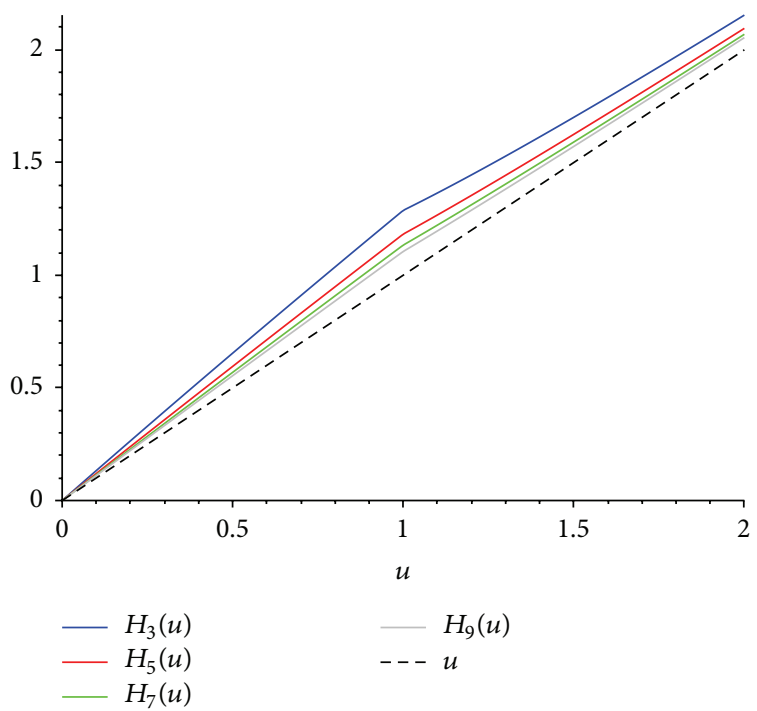

Figure 2: Example of kernels $H_{w}(u), w=3,5,7,9$.

uniformly with respect to every (not trivial) bounded interval $J \subset \mathbb{R}$; that is, for every $\varepsilon>0$ there exists $\bar{w}>0$ such that $V_{J}\left[G_{w}\right] / m(J)<\varepsilon$, for every $w \geq \bar{w}$ and for every bounded interval $J \subset \mathbb{R}$.

Remark 27. We point out that Assumption $\left(\bar{K}_{w} .3\right)$ is due to the nonlinear frame and of course it is obviously satisfied in the linear case $\left(H_{w}(u)=u, u \in \mathbb{R}\right)$. Moreover it is not difficult to provide examples of kernels which fulfill all the previous assumptions. For example, we can consider the kernel functions $\bar{K}_{w}(t, u)=L_{w}(t) H_{w}(u), t \in \mathbb{R}_{0}^{+}, u \in \mathbb{R}, w>$ 0 , where $\left\{L_{w}\right\}_{w>0}$ are approximate identities,

$$
H_{w}(u)= \begin{cases}u+\log \left(1+\frac{u}{w}\right), & 0 \leq u<1, \\ u+\log \left(1+\frac{1}{w u}\right), & u \geq 1,\end{cases}
$$

and the definition of $H_{w}(u)$ is extended in odd way for $u<0$ (see Figure 2).

The problem of the convergence in variation for the nonlinear integral operators (III) was faced in $[58,59]$; in particular, the main convergence result reads as follows.

Theorem 28 (see [58]). If $f \in A C\left(\mathbb{R}^{N}\right)$ and $\left\{\bar{K}_{w}\right\}_{w>0}$ satisfy $\left(\bar{K}_{w}, i\right), i=1,2,3$, then

$$
\lim _{w \rightarrow+\infty} V_{\mathbb{R}^{N}}\left[\bar{T}_{w} f-f\right]=0 .
$$

Similar approximation results were obtained in [60] for the nonlinear convolution integral operators (III) in the frame of $\mathrm{BV}^{\varphi}\left(\mathbb{R}^{N}\right)$ and in [61] for the nonlinear version of the Mellin integral operators (II) in $\mathrm{BV}\left(\mathbb{R}_{+}^{N}\right)$.

We finally point out that, besides the problem of convergence, the rate of approximation has been also studied in all the previously mentioned settings. In order to do it, as it is natural, one has to introduce suitable Lipschitz classes 
which take into account the variation functional. We point out that, in order to approach the mentioned problem, the assumptions on kernels have to be slightly modified.

For example, let us consider the case of the convolution integral operators (I) in the setting of $\mathrm{BV}\left(\mathbb{R}^{N}\right)$. In this frame the Lipschitz class is defined as

$$
\begin{aligned}
& V \operatorname{Lip}^{N}(\alpha):=\left\{f \in \operatorname{BV}\left(\mathbb{R}^{N}\right): V_{\mathbb{R}^{N}}\left[\tau_{\mathrm{t}} f-f\right]\right. \\
& \left.\quad=O\left(|\mathrm{t}|^{\alpha}\right), \text { as }|\mathrm{t}| \longrightarrow 0\right\},
\end{aligned}
$$

$\alpha>0$, and Assumption $\left(K_{w}, 2\right)$ has to be replaced by the following:

$$
\left(K_{w}^{\prime} .2\right) \text { for any fixed } \delta>0, \int_{|t|>\delta}\left|L_{w}(\mathrm{t})\right| d t=O\left(w^{-\alpha}\right),
$$
as $w \rightarrow+\infty$.

Moreover we will say that $\left\{K_{w}\right\}_{w>0}$ is an $\alpha$-singular kernel, for $0<\alpha \leq 1$, if

$$
\int_{|\mathrm{t}|>\delta}\left|K_{w}(\mathrm{t})\right| d \mathrm{t}=O\left(w^{-\alpha}\right), \quad \text { as } w \longrightarrow+\infty,
$$

for every $\delta>0$. Then it is possible to obtain the following result about the order of approximation for the convolution integral operators (I).

Theorem 29 (see [25]). Let $f \in V \operatorname{Lip}^{N}(\alpha)$ and let $\left\{K_{w}\right\}_{w>0} \subset$ $\mathscr{K}_{w}$ be an $\alpha$-singular kernel satisfying $\left(K_{w} .1\right)$ and $\left(K_{w}^{\prime} .2\right)$. Moreover assume that there exists $0<\widetilde{\delta}<1$ such that

$$
\int_{|\mathrm{t}| \leq \tilde{\delta}}\left|K_{w}(\mathrm{t})\right||\mathrm{t}|^{\alpha} d \mathrm{t}=O\left(w^{-\alpha}\right) \text {, as } w \longrightarrow+\infty .
$$

Then

$$
V_{\mathbb{R}^{N}}\left[T_{w} f-f\right]=O\left(w^{-\alpha}\right),
$$

as $w \rightarrow+\infty$.

Similar results in the nonlinear case were obtained in [58], while, for results about the rate of approximation for convolution integral operators with respect to the multidimensional $\varphi$-variation, see [21] and [60] (nonlinear case).

The case of Mellin integral operators was studied in [36] and in [61] (nonlinear case) with respect to the Tonelli variation, while the case of the multidimensional $\varphi$-variation was studied in [56]. We finally refer to $[62,63]$ for approximation results in the slightly different setting of $\mathrm{BV}^{\varphi}\left(\left(\mathbb{R}_{0}^{+}\right)^{N}\right)$.

\section{Competing Interests}

The authors declare that there is no conflict of interests regarding the publication of this article.

\section{Acknowledgments}

The authors are Members of the Gruppo Nazionale per l'Analisi Matematica, la Probabilitá e le loro Applicazioni (GNAMPA) of the Istituto Nazionale di Alta Matematica
(INdAM). This work was supported by Department of Mathematics and Computer Sciences, University of Perugia. Moreover, the first author of the paper has been partially supported within the GNAMPA-INdAM Project 2016 "Problemi di Regolarità nel Calcolo delle Variazioni e di Approssimazione."

\section{References}

[1] R. G. Mamedov, The Mellin Transform and Approximation Theory, Elm, Baku, 1991 (Russian).

[2] P. L. Butzer and S. Jansche, "A direct approach to the Mellin transform," Journal of Fourier Analysis and Applications, vol. 3, no. 4, pp. 325-376, 1997.

[3] W. Z. Chen and S. S. Guo, "On the rate of convergence of the gamma operator for functions of bounded variation," Approximation Theory and its Applications, vol. 1, no. 5, pp. 85-96, 1985.

[4] C. Bardaro and G. Vinti, "Modular estimates of integral operators with homogeneous kernels in Orlicz type classes," Results in Mathematics, vol. 19, no. 1-2, pp. 46-53, 1991.

[5] I. Mantellini and G. Vinti, "Modular estimates for nonlinear integral operators and applications in fractional calculus," Numerical Functional Analysis and Optimization, vol. 17, no. 1-2, pp. 143-165, 1996.

[6] C. Bardaro, J. Musielak, and G. Vinti, Nonlinear Integral Operators and Applications, vol. 9 of De Gruyter Series in Nonlinear Analysis and Applications, Walter de Gruyter, New York, NY, USA, 2003.

[7] G. Vinti, "Approximation in Orlicz spaces for linear integral operators and Applications," Rendiconti del Circolo Matematico di Palermo, supplement 76, no. 2, pp. 103-127, 2005.

[8] H. Karsli, "Rate of convergence of new Gamma type operators for functions with derivatives of bounded variation," Mathematical and Computer Modelling, vol. 45, no. 5-6, pp. 617-624, 2007.

[9] C. Bardaro, G. Vinti, and H. Karsli, "On pointwise convergence of linear integral operators with homogeneous kernels," Integral Transforms and Special Functions, vol. 19, no. 5-6, pp. 429-439, 2008.

[10] H. Karsli, V. Gupta, and A. Izgi, "Rate of pointwise convergence of a new kind of gamma operators for functions of bounded variation," Applied Mathematics Letters, vol. 22, no. 4, pp. 505$510,2009$.

[11] L. Angeloni and G. Vinti, "Approximation with respect to Goffman-Serrin variation by means of non-convolution type integral operators," Numerical Functional Analysis and Optimization, vol. 31, no. 4-6, pp. 519-548, 2010.

[12] C. Bardaro, G. Vinti, and H. Karsli, "Nonlinear integral operators with homogeneous kernels: pointwise approximation theorems," Applicable Analysis, vol. 90, no. 3-4, pp. 463-474, 2011.

[13] A. Boccuto, D. Candeloro, and A. R. Sambucini, "Vitali-type theorems for filter convergence related to vector lattice-valued modulars and applications to stochastic processes," Journal of Mathematical Analysis and Applications, vol. 419, no. 2, pp. 818838, 2014.

[14] V. Gupta and R. P. Agarwal, Convergence Estimates in Approximation Theory, Springer, 2014.

[15] G. Vinti and L. Zampogni, "Approximation results for a general class of Kantorovich type operators," Advanced Nonlinear Studies, vol. 14, no. 4, pp. 991-1011, 2014. 
[16] A. Boccuto, D. Candeloro, and A. R. Sambucini, " $L^{P}$ spaces in vector lattices and applications," Mathematica Slovaca, https:// arxiv.org/abs/1604.07570.

[17] M. Bertero and E. R. Pike, "Exponential-sampling method for Laplace and other dilationally invariant transforms: I. Singular-system analysis. II. Examples in photon correlation spectroscopy and Fraunhofer diffraction," Inverse Problems, vol. 7, pp. 1-41, 1991.

[18] P. L. Butzer and S. Jansche, “The exponential sampling theorem of signal analysis," Atti del Seminario Matematico e Fisico dell'Università di Modena e Reggio Emilia, vol. 46, pp. 99-122, A Special Issue of the International Conference in Honour of Prof. Calogero Vinti, 1998.

[19] C. Bardaro, P. L. Butzer, and I. Mantellini, "The exponential sampling theorem of signal analysis and the reproducing kernel formula in the Mellin transform setting," Sampling Theory in Signal and Image Processing, vol. 13, no. 1, pp. 35-66, 2014.

[20] C. Bardaro, P. L. Butzer, and I. Mantellini, "The Mellin-Parseval formula and its interconnections with the exponential sampling theorem of optical physics," Integral Transforms and Special Functions, vol. 27, no. 1, pp. 17-29, 2016.

[21] L. Angeloni and G. Vinti, "Convergence and rate of approximation for linear integral operators in $B V^{\varphi}$-spaces in multidimensional setting," Journal of Mathematical Analysis and Applications, vol. 349, no. 2, pp. 317-334, 2009.

[22] J. Musielak and W. Orlicz, "On generalized variations (I)," Studia Mathematica, vol. 18, pp. 11-41, 1959.

[23] I. Mantellini and G. Vinti, " $\Phi$-variation and nonlinear integral operators," in Atti del Seminario Matematico e Fisico dell' Università di Modena e Reggio Emilia, vol. 46 of International Conference in Honour of Prof. Calogero Vinti, pp. 847-862, 1998.

[24] S. Sciamannini and G. Vinti, "Convergence and rate of approximation in $B V^{\varphi}(\mathbb{R})$ for a class of integral operators," Approximation Theory and its Applications, vol. 17, pp. 17-35, 2001.

[25] C. Bardaro, P. L. Butzer, R. L. Stens, and G. Vinti, "Convergence in variation and rates of approximation for Bernstein-type polynomials and singular convolution integrals," Analysis, vol. 23, no. 4, pp. 299-340, 2003.

[26] L. Angeloni and G. Vinti, "Approximation by means of nonlinear integral operators in the space of functions with bounded $\varphi$-variation," Differential and Integral Equations, vol. 20, no. 3, pp. 339-360, 2007.

[27] L. Angeloni and G. Vinti, "Errata Corrige to: approximation by means of nonlinear integral operators in the space of functions with bounded $\varphi$-variation," Differential and Integral Equations, vol. 23, no. 7-8, pp. 795-799, 2010.

[28] L. Tonelli, "Su alcuni concetti dell'analisi moderna," Annali della Scuola Normale Superiore di Pisa, vol. 11, no. 2, pp. 107-118, 1942.

[29] T. Radó, Length and Area, American Mathematical Society Colloquium Publications, vol. 30, American Mathematical Society, New York, NY, USA, 1948.

[30] C. Vinti, "Perimetro-variazione," Annali della Scuola Normale Superiore di Pisa, vol. 18, no. 3, pp. 201-231, 1964.

[31] L. Cesari, "Sulle funzioni a variazione limitata," Annali della Scuola Normale Superiore di Pisa, vol. 5, pp. 299-313, 1936.

[32] K. Krickeberg, "Distributionen, Funktionen beschränkter Variation und Lebesguescher Inhalt nichtparametrischer Flächen," Annali di Matematica Pura ed Applicata. Serie Quarta, vol. 44, no. 1, pp. 105-133, 1957.

[33] E. De Giorgi, "Su una teoria generale della misura $(r-1)$ dimensionale in uno spazio ad $r$ dimensioni," Annali di Matematica Pura ed Applicata, vol. 36, no. 1, pp. 191-213, 1954.
[34] E. Giusti, Minimal Surfaces and Functions of Bounded Variation, vol. 80 of Monographs in Mathematics, Birkhäuser, Basel, Switzerland, 1984.

[35] J. Serrin, "On the differentiability of functions of several variables," Archive for Rational Mechanics and Analysis, vol. 7, pp. 359-372, 1961.

[36] L. Angeloni and G. Vinti, "Variation and approximation in multidimensional setting for Mellin integral operators," in New Perspectives on Approximation and Sampling Theory: Festschrift in Honor of Paul Butzer's 85th Birthday, pp. 299-317, Birkhauser, 2014.

[37] C. Jordan, "Sur la serie de Fourier," Comptes Rendus de l'Académie des Sciences Paris, vol. 92, pp. 228-230, 1881.

[38] N. Wiener, "The quadratic variation of a function and its fourier coefficients," Journal of Mathematics and Physics, vol. 3, no. 2, pp. 72-94, 1924.

[39] L. C. Young, "An inequality of the Hölder type, connected with Stieltjes integration," Acta Mathematica, vol. 67, no. 1, pp. 251282, 1936.

[40] E. R. Love and L. C. Young, "Sur une classe de fonctionnelles linéaires," Fundamenta Mathematicae, vol. 28, no. 1, pp. 243-257, 1937.

[41] L. C. Young, "Sur une généralisation de la notion de variation de puissance $p^{\text {ieme }}$ bornée au sens de M. Wiener, et sur la convergence des séries de Fourier," Comptes Rendus de l'Académie des Sciences Paris, vol. 204, pp. 470-472, 1937.

[42] J. Musielak, Orlicz Spaces and Modular Spaces, vol. 1034 of Lecture Notes in Mathematics, Springer, Berlin, Germany, 1983.

[43] J. Szelmeczka, "On convergence of singular integrals in the generalized variation metric," Functiones et Approximatio, Commentarii Mathematici, vol. 15, pp. 53-58, 1986.

[44] W. Matuszewska and W. Orlicz, "On property B1 for functions of bounded $\varphi$-variation," Bulletin of the Polish Academy of Sciences Mathematics, vol. 35, no. 1-2, pp. 57-69, 1987.

[45] A.-R. K. Ramazanov, "On approximation of functions in terms of $\Phi$-variation," Analysis Mathematica, vol. 20, no. 4, pp. 263281, 1994.

[46] J. A. Adell and J. de la Cal, "Bernstein-type operators diminish the $\varphi$-variation," Constructive Approximation, vol. 12, no. 4, pp. 489-507, 1996.

[47] V. V. Chistyakov and O. E. Galkin, "Mappings of bounded $\phi$ variation with arbitrary function $\phi$," Journal of Dynamical and Control Systems, vol. 4, no. 2, pp. 217-247, 1998.

[48] J. Appell, J. Banaś, and N. Merentes, Bounded Variation and Around, vol. 17 of De Gruyter Series in Nonlinear Analysis and Applications, De Gruyter, Berlin, Germany, 2014.

[49] J. Musielak, "Sequences of bounded $\varphi$-variation and weighted unconditional convergence of series," Demonstratio Mathematica, vol. 38, no. 1, pp. 153-162, 2005.

[50] G. Vinti, "The generalized $\varphi$-variation in the sense of vitali: estimates for integral operators and applications in fractional calculus," Commentationes mathematicae: Prace matematyczne, vol. 34, pp. 199-213, 1994.

[51] L. Angeloni and G. Vinti, "A sufficient condition for the convergence of a certain modulus of smoothness in multidimensional setting," Communications on Applied Nonlinear Analysis, vol. 20, no. 1, pp. 1-20, 2013.

[52] P. L. Butzer and R. J. Nessel, Fourier Analysis and Approximation I, Academic Press, New York, NY, USA, 1971.

[53] J. Musielak, "Nonlinear approximation in some modular function spaces. I," Mathematica Japonica, vol. 38, no. 1, pp. 83-90, 1993. 
[54] L. Angeloni, "A characterization of a modulus of smoothness in multidimensional setting," Bollettino della Unione Matematica Italiana. Serie IX, vol. 4, no. 1, pp. 79-108, 2011.

[55] L. Angeloni and G. Vinti, "A characterization of absolute continuity by means of Mellin integral operators," Zeitschrift für Analysis und Ihre Anwendungen, vol. 34, no. 3, pp. 343-356, 2015.

[56] L. Angeloni and G. Vinti, "Convergence and rate of approximation in $B V^{\varphi}\left(\mathbb{R}_{+}^{N}\right)$ for a class of Mellin integral operators," Atti della Accademia Nazionale dei Lincei-Rendiconti Lincei: Matematica e Applicazioni, vol. 25, pp. 217-232, 2014.

[57] L. Angeloni and G. Vinti, "A concept of absolute continuity and its characterization in terms of convergence in variation," Mathematische Nachrichten, 2016.

[58] L. Angeloni and G. Vinti, "Convergence in variation and rate of approximation for nonlinear integral operators of convolution type," Results in Mathematics, vol. 49, no. 1-2, pp. 1-23, 2006.

[59] L. Angeloni and G. Vinti, "Erratum to: convergence in variation and rate of approximation for nonlinear integral operators of convolution type," Results in Mathematics, vol. 57, no. 3-4, pp. 387-391, 2010.

[60] L. Angeloni, "Approximation results with respect to multidimensional $\varphi$-variation for nonlinear integral operators," Zeitschrift für Analysis und Ihre Anwendungen, vol. 32, no. 1, pp. 103128, 2013.

[61] L. Angeloni and G. Vinti, "Convergence in variation and a characterization of the absolute continuity," Integral Transforms and Special Functions, vol. 26, no. 10, pp. 829-844, 2015.

[62] L. Angeloni, "Convergence in variation for a homothetic modulus of smoothness in multidimensional setting," Communications on Applied Nonlinear Analysis, vol. 19, no. 1, pp. 1-22, 2012.

[63] L. Angeloni and G. Vinti, "Approximation in variation by homothetic operators in multidimensional setting," Differential and Integral Equations, vol. 26, no. 5-6, pp. 655-674, 2013. 


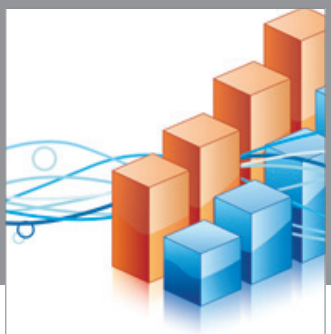

Advances in

Operations Research

vatem alat4

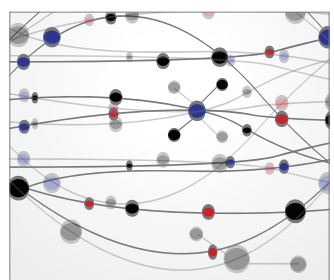

\section{The Scientific} World Journal
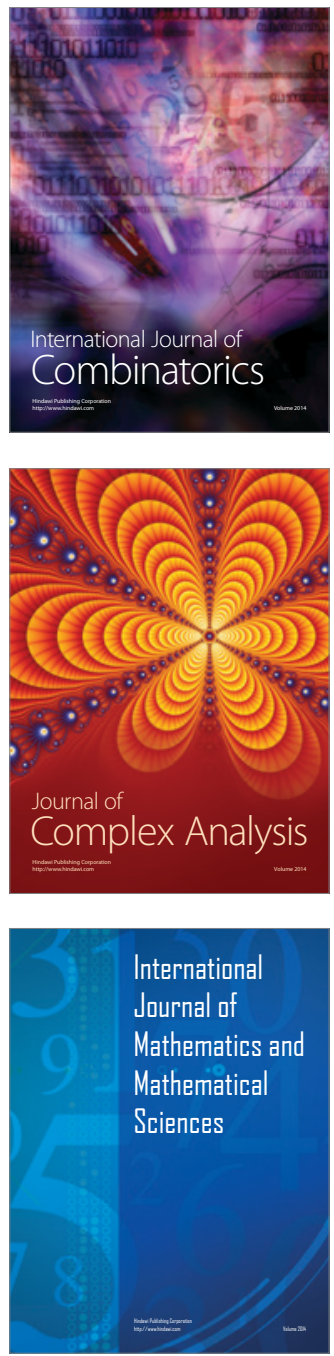
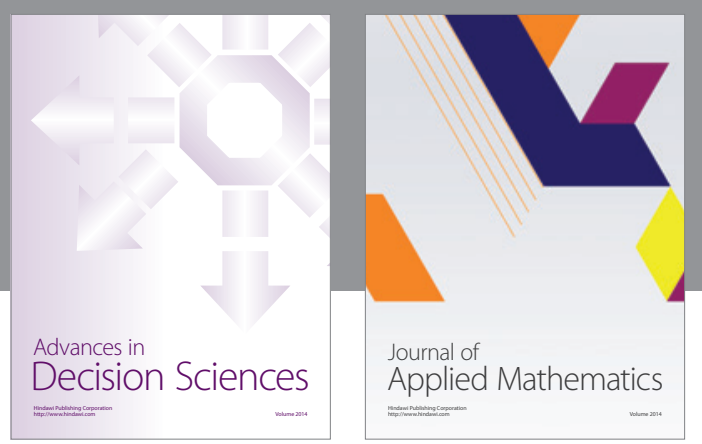

Algebra

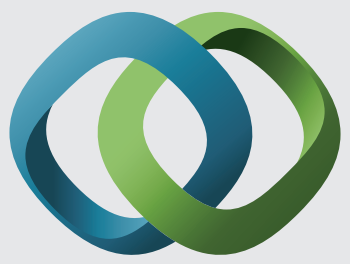

\section{Hindawi}

Submit your manuscripts at

http://www.hindawi.com
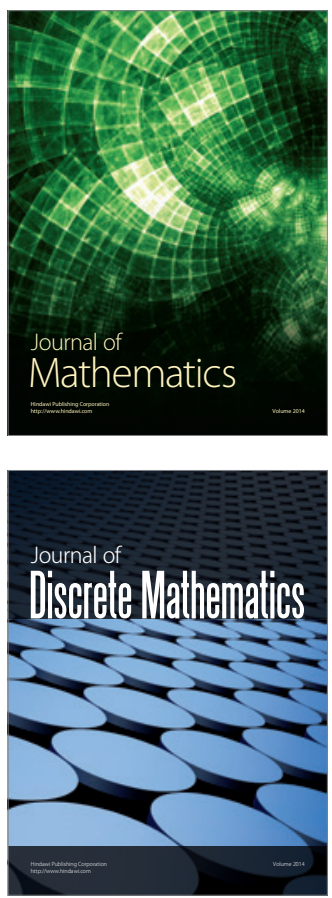

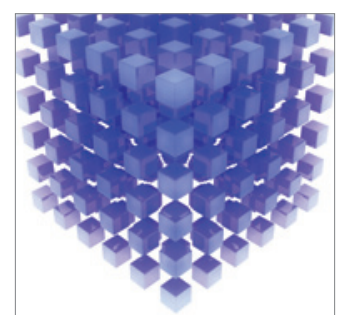

Mathematical Problems in Engineering
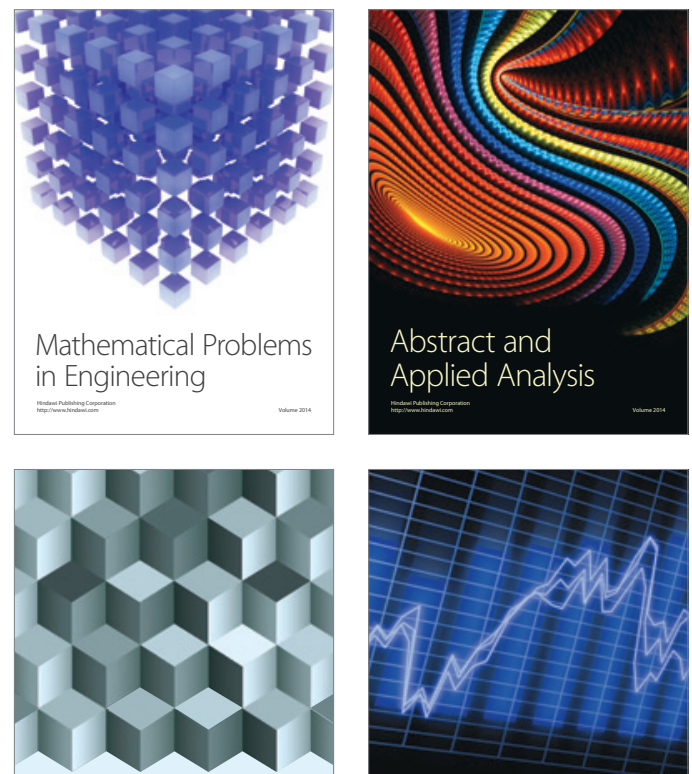

Journal of

Function Spaces

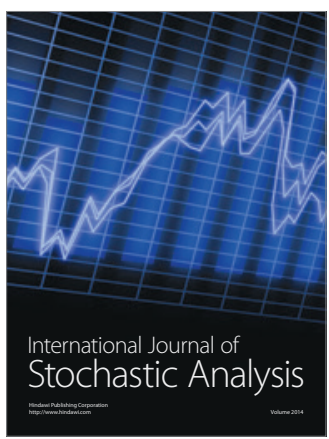

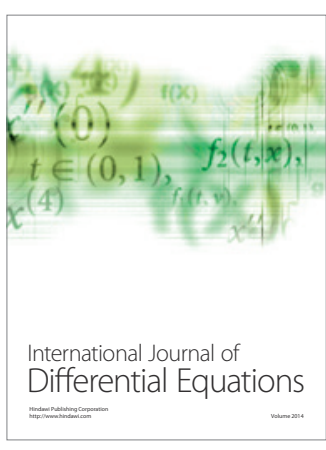
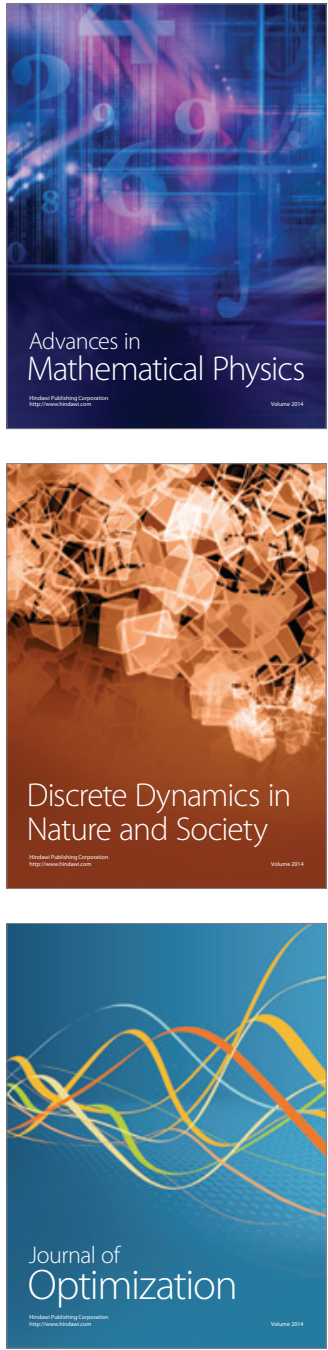